\title{
Characterization and chromosomal localization of JAM-1, a platelet receptor for a stimulatory monoclonal antibody
}

\author{
Ulhas P. Naik ${ }^{1, \star}$, Meghna U. Naik ${ }^{1}$, Kristin Eckfeld ${ }^{1}$, Patricia Martin-DeLeon ${ }^{1}$ and Jozef Spychala ${ }^{2}$ \\ ${ }^{1}$ Department of Biological Sciences, University of Delaware, Newark, DE 19716, USA \\ 2Department of Pharmacology, The University of North Carolina at Chapel Hill, Chapel Hill, NC, USA \\ *Author for correspondence (e-mail: unaik@udel.edu) \\ Accepted 20 November 2000 \\ Journal of Cell Science 114, 539-547 (C) The Company of Biologists Ltd
}

\section{SUMMARY}

We have previously reported the purification and characterization of a $32 \mathrm{kDa}$ platelet surface glycoprotein that is recognized by the stimulatory monoclonal antibody, F11. The cDNA has been cloned and found to encode the human homolog of the murine junctional adhesion molecule, JAM; we therefore named this human homolog JAM-1. Northern blot analysis indicated that JAM-1 mRNA is expressed as multiple species, the predominant transcript being $\sim 4.0 \mathrm{~kb}$ in size. Genetic mapping analysis using fluorescence in situ hybridization (FISH) showed that it is localized to chromosome 1q21.1-21.3. Recombinant JAM-1, when expressed in Chinese hamster ovary (CHO) cells, localized to the cell membrane with intense staining where two adjacent cells actually made contact with each other, suggesting that, similar to murine JAM, human JAM-1 may also localize at the cell-cell junction. In wellspread cells, JAM-1 co-localized with F-actin at the cell-cell contacts and at the membrane ruffles, but not at the stress fibers. Interestingly, JAM-1 localizes only to the cell-cell junctions formed by two transfected cells and not to the cell-cell junctions formed by a transfected cell with an untransfected cell, suggesting that JAM-1 may facilitate cell adhesion through homophilic binding. In addition, human platelets specifically bind to a monolayer of $\mathrm{CHO}$ cells expressing human JAM-1, further supporting homophilic interactions. The results presented here indicate that JAM-1, a receptor for a platelet-activating antibody, is the human homolog of the junctional adhesion molecule. JAM-1 is a single copy gene, which is constitutively expressed on various tissues and cells, and may be involved in cell to cell adhesion through homophilic interaction.

Key words: JAM-1, CAM, Platelet, Activation, Adhesion, Homophilic

\section{INTRODUCTION}

Following tissue injury and vessel wall stimulation, a number of events are triggered to recruit circulating blood cells to specific sites, including platelet activation, endothelial cell stimulation, and leukocyte transmigration through the endothelium to mediate the host defense mechanisms of inflammation, thrombosis and wound healing (Celi et al., 1997; Etzioni, 1996). These processes are highly regulated and involve various cell adhesion molecules expressed by both the endothelium and circulating blood cells. The involved cell adhesion molecules belong to four major superfamilies: integrins, immunoglobulin (Ig)-like proteins (CAMs), selectins and cadherins (Rosales et al., 1995). Leukocytes constitutively express $\alpha 2$ integrins; integrin $\alpha_{\operatorname{IIb}} \beta_{3}$ is the major platelet integrin, whereas integrin $\alpha_{v} \beta_{3}$ is predominantly expressed on endothelial cells. CAMs are expressed on endothelial cells, leukocytes and platelets, and serve as counter receptors/ligands for integrins. These interactions between integrins and Ig-like CAMs play an important role in leukocyte transmigration through the endothelium (Vaporciyan et al., 1993; Wahl et al., 1996).

Cytokines released at the inflammatory site induce the expression of various CAMs on the endothelium. Leukocytes adhere to these molecules through their surface integrins, which accounts in part for the recruitment of leukocytes during an inflammatory or immune reaction (Chiba et al., 1999). Leukocyte adhesion to endothelial cells triggers intracellular signals that in turn, lead to the opening of the junctions between neighboring endothelial cells, facilitating leukocyte transmigration (Celi et al., 1997). The mechanism involved in the opening of the junction during leukocyte transmigration is not yet known. It has been suggested that phosphorylation of certain junctional proteins may regulate this process (Dejana et al., 1995; Takaishi et al., 1997). Recently, a murine Ig superfamily molecule, JAM, has been identified (MartinPadura et al., 1998). This murine JAM is expressed at cell-cell junctions in endothelial and epithelial cells, and has been implicated in monocyte transmigration (Del Maschio et al., 1999). Subsequently, Ozaki and colleagues reported the identification of human and bovine homologs of JAM and showed that it redistributes from the cell-cell junction to the cell surface upon activation of endothelial cells by combined treatment with TNF- $\alpha$ and IFN- $\gamma$ (Ozaki et al., 1999).

Monoclonal antibody $\mathrm{F} 11$ is a potent platelet agonist (Kornecki et al., 1990), as it induces platelet granular secretion 
and platelet aggregation. F11 recognizes a $32 \mathrm{kDa}$ glycoprotein as an antigen and crosslinks it to the Fc $\gamma \mathrm{RII}$ receptor. As a result of this crosslinking, a signaling cascade is activated that involves a rise in intracellular $\mathrm{Ca}^{2+}$, activation of protein kinase $\mathrm{C}$, intracellular protein phosphorylation and activation of integrin $\alpha_{\text {IIb }} \beta_{3}$, the fibrinogen receptor (Naik et al., 1995; Wang et al., 1995). F11 also potentiates the effect of other physiological agonists in the absence of Fc $\gamma \mathrm{RII}$ receptor engagement. We have previously reported the purification of the F11 antigen from human platelets (Naik et al., 1995). Here we report the cloning, cellular expression and chromosomal localization of the F11 antigen gene, which has also been independently identified elsewhere (Sobocka et al., 2000). Amino acid analysis indicates that the F11 antigen is a member of the Ig-superfamily of cell adhesion molecules. The deduced amino acid sequence of the F11 antigen has about 69\% identity to the murine junctional adhesion molecule (JAM) and is identical to its human homolog. We therefore named the F11 antigen, the human homolog of JAM, JAM-1.

\section{MATERIALS AND METHODS}

Geneticin (G418), Lipofectamine and tissue culture media were purchased from Gibco-BRL (Gaithersburg, MD). Unless specified otherwise, all other chemicals were of analytical grade purchased from Sigma (St Louis, MO).

\section{cDNA cloning of JAM-1}

Two degenerate primers, sense $5^{\prime}-\mathrm{AT}(\mathrm{T} / \mathrm{C} / \mathrm{A}) \mathrm{CC}(\mathrm{T} / \mathrm{C} / \mathrm{A} / \mathrm{G}) \mathrm{GA}(\mathrm{A} / \mathrm{G})$ $\mathrm{AA}(\mathrm{T} / \mathrm{C}) \mathrm{AA}(\mathrm{T} / \mathrm{C}) \mathrm{CC}-3^{\prime}$ and antisense $5^{\prime}-(\mathrm{T} / \mathrm{C}) \mathrm{TT}(\mathrm{A} / \mathrm{G}) \mathrm{AA}(-$ G/A/T/C)GT(A/G/T)AT(T/C/G/A)CC-3', corresponding to the Nterminal and the internal peptide sequence of F11 antigen/JAM-1, respectively (Naik et al., 1995), were synthesized and used in RT-PCR reactions using total RNA from freshly isolated human platelets. The PCR product obtained was directly subcloned into the PCR 2.1 vector (Clontech) by TA-TOPO cloning. Sequencing and GenBank searching revealed several partial ESTs. EST AA101561 was obtained from the American Type Culture Collection (ATCC, Manassas, VA) and used to screen a human liver cDNA library (Clontech) using the described procedure (Spychala et al., 1996). Consequently, several positive clones were obtained, one of which was a full-length cDNA. The sequence has been deposited in the GenBank database under the Accession Number AF172398.

\section{Construction of expression plasmids}

A hemoagglutinin (HA) epitope (AYPYDVPDYA) was inserted between the signal peptide and the first Ig domain of JAM-1 using PCR. The resultant cDNA was directionally cloned into the mammalian expression vector pcDNA3.1, and the sequence of the resulting clones was verified using automated sequencing. This epitope-tagging allowed us to follow the expression of recombinant JAM-1, and distinguish it from endogenous JAM-1.

\section{Antibodies}

FITC-conjugated phalloidin was purchased from Molecular Probes (Eugene, OR). TRITC-labeled anti-mouse immunoglobulin G (IgG) was purchased from Jackson ImmunoResearch Laboratories (West Grove, PA). Mouse monoclonal antibody against the HA-tag was purchased from Babco (Richmond, CA). HRP-conjugated anti-mouse and anti-rabbit antibodies were purchased from Amersham Pharmacia Biotech (Piscataway, NJ).

\section{Cell culture}

Chinese hamster ovary (CHO) and human breast cancer cells (T47D) were obtained from ATCC. CHO cells were maintained in DMEM supplemented with $10 \%$ fetal bovine serum (FBS), 100 units/ml of penicillin and $100 \mu \mathrm{g} / \mathrm{ml}$ streptomycin. T47D cells were maintained in RPMI-1640 supplemented with $10 \%$ FBS, $10 \mu \mathrm{g} / \mathrm{ml}$ insulin, 100 units/ml penicillin and $100 \mu \mathrm{g} / \mathrm{ml}$ streptomycin. All cells were cultured in a humidified atmosphere of $5 \% \mathrm{CO}_{2}$ in air.

\section{Transfections}

Both CHO and T47D cell lines were transfected using Lipofectamine (GibcoBRL) following the manufacturer's protocol. Control cells were transfected with an empty pcDNA plasmid. G418 was added to the culture medium 24 hours after transfection at a final concentration of $500 \mu \mathrm{g} / \mathrm{ml}$, and the resistant individual colonies were isolated to obtain single cell clones, or expanded as pools of stably transfected cells. The expression of JAM-1 was determined by immunoblotting. All the cells were thereafter maintained in culture medium containing $300 \mu \mathrm{g} / \mathrm{ml}$ of $\mathrm{G} 418$.

\section{Immunofluorescence}

JAM-1-overexpressing CHO cells were cultured in Lab-TekII 8chambered coverglass slides (Nunc, Naperville, IL), fixed in freshly prepared $4 \%$ paraformaldehyde for 10 minutes, permeabilized in $0.2 \%$ Triton X-100 for 5 minutes, and blocked with phosphate buffered saline (PBS) containing 3\% BSA (blocking solution) for 1 hour. Cells were then incubated with mouse anti-HA antibody (1:150) overnight at $4^{\circ} \mathrm{C}$. After incubation, cells were washed three times with blocking solution and incubated with TRITC-labeled Donkey anti-Mouse IgG secondary antibody (1:200) for 1 hour at room temperature. For staining of filamentous actin, FITC-conjugated phalloidin $(1: 100)$ was included during incubation along with the secondary antibody. Cells were washed three times in blocking solution with a final wash of PBS. Slowfade (Molecular Probes) was added to minimize fading of the fluorescence intensity. Confocal microscopy was performed using a Zeiss LSM510 laser-scanning microscope.

\section{FISH analysis}

The JAM-1 cDNA clone was biotinylated by nick translation and used as a probe in FISH studies as described previously, with some modifications (Deng et al., 1997; Nagle et al., 1994). Approximately $400 \mathrm{ng}$ of the biotin-labeled cDNA was co-precipitated with sonicated herring sperm DNA and dissolved in hybridization solution [50\% $(\mathrm{v} / \mathrm{v})$ formamide, $2 \times \mathrm{SSC}$ and $10 \%(\mathrm{w} / \mathrm{v})$ dextran sulfate (Hybrisol VII, Oncor, Gaithersburg, MD)]. The probe was then denatured for 8 minutes at $75^{\circ} \mathrm{C}$ and immediately applied to human metaphase spreads prepared from peripheral lymphocyte cultures for overnight hybridization. Post-hybridization washes were brief ( 2 minutes) and carried out at $37^{\circ} \mathrm{C}$. The detection and amplification of the signal was performed with FITC-labeled avidin and anti-avidin (Oncor) and chromosomes were counterstained with diaminophenylinodole (DAPI) in p-phenylenediamine $(0.1 \mu \mathrm{g} / \mathrm{ml})$ for microscopic examination. The slides were viewed with a Zeiss Axioskop equipped with a Sensys cooled CCD camera (Photometrics, Tucson, AZ). Image capture was performed with software from IPLab Spectrum Module (Scanalytics, Fairfax, VA). Separate images of DAPI-banded chromosomes and FITC hybridization signals were acquired and electronically superimposed.

\section{Northen blot analysis}

Multiple tissue northern (MTN) blots were purchased from Clontech. An EcoRI fragment ( $\sim 800 \mathrm{bp})$ of JAM-1, which includes only the coding region, was used as a probe. The northern blots were washed with stringent conditions according to the manufacturer's instructions. A $\beta$-actin probe was used to test for equal loading of the mRNA.

\section{RT-PCR}

Total RNA was isolated from K562 cells using TRIZOL reagent (GibcoBRL). Poly(A) ${ }^{+}$RNA was isolated using an oligo(dT) cellulose 
column, following the manufacturer's protocol. A reverse transcriptase reaction was carried out for 30 minutes at $50^{\circ} \mathrm{C}$ using 1 $\mu \mathrm{g}$ of total RNA or $0.1 \mu \mathrm{g}$ of poly $(\mathrm{A})^{+}$RNA per reaction. The first 10 cycles of PCR amplification were carried out at $94^{\circ} \mathrm{C}$ for 30 seconds, $45^{\circ} \mathrm{C}$ for 30 seconds, $68^{\circ} \mathrm{C}$ for 2 minutes, and during the next 20 cycles, the elongation time was extended to 3 minutes. Various primers derived from JAM-1 cDNA as indicated (see Fig. 3A) were used to amplify specific size products. PCR products were analyzed using agarose gel electrophoresis.

\section{Platelet adhesion}

Platelets were isolated from healthy donors by venipuncture using sodium citrate as an anticoagulant (Naik et al., 1991). Platelet-rich plasma (PRP) was prepared by centrifugation at $200 \boldsymbol{g}$ for 10 minutes. PRP was diluted with PBS to $1 \times 10^{6}$ platelets $/ \mathrm{ml}$ and $200 \mu \mathrm{l}$ of this platelet suspension was added to the cover glass chambers pre-plated with a monolayer of $\mathrm{CHO}$ cells mock transfected or transfected with JAM-1. After 30 minutes of incubation at room temperature, the chambers were washed three times and then incubated for 10 minutes with FITClabeled cyclic RGD peptide XL086 (Mousa et al., 2000). This peptide specifically binds to the platelet-specific surface integrin $\alpha_{\mathrm{IIb}} \beta_{3}$ and can be used to detect bound platelets (Mousa et al., 2000). The number of platelets bound to the monolayer was quantified by counting randomly at $100 \times$ magnification using confocal laser microscopy.

\section{RESULTS}

\section{cDNA cloning of JAM-1}

We have previously reported the purification and partial amino acid sequence of the platelet surface protein recognized by the stimulatory antibody F11 (Naik et al., 1995). To clone this platelet surface protein, degenerate primers derived from $\mathrm{N}$-terminal and internal amino acid sequences of F11 antigen were used in RT-PCR reactions. The amplified PCR product was cloned into the pCR 2.1 vector and sequenced. A GenBank database search using this cDNA sequence revealed several partial EST entries. We obtained an EST clone from ATCC and used the cDNA insert as a probe to screen a human liver library. We isolated a 1.9 $\mathrm{kb}$ cDNA clone of the F11 antigen containing a $900 \mathrm{bp}$ open reading frame (Fig. 1A). The deduced amino acid sequence of the F11 antigen is $\sim 69 \%$ identical to the recently identified mouse junctional cell adhesion molecule, JAM (Fig. 1B) (Martin-Padura et al., 1998), and identical to the human homolog of JAM termed JAM-1 (Ozaki et al., 1999). We therefore conclude that the F11 antigen is JAM-1. The cDNA encodes the 299 amino acid JAM-1 precursor containing a 27 amino acid plasma membrane localization signal peptide. The predicted signal peptide cleavage site corresponds to the $\mathrm{N}$-terminal sequence of the native mature protein. JAM-1 is a type one integral membrane protein with a single

B transmembrane domain of 19 hydrophobic residues (V237V254), and also shows significant similarity to other cell adhesion molecules such as VCAM-1 and NCAM. The extracellular domain has two C2-type disulfide-linked Ig-like domains; JAM-1 also has a short, 42-amino acid cytoplasmic tail. The protein sequence of JAM-1 contains two putative Nglycosylation sites (N185 and N191) on its external surface and several consensus phosphorylation sites for PKC cAMPdependent protein kinase A (PKA) and casein kinase II within the cytoplasmic domain. In addition, the cytoplasmic domain contains two tyrosine residues separated by 18 amino acid residues in consensus for tyrosine phosphorylation and binding to the SH-2 domain (Jackson et al., 1997a). VE-JAM, another junctional adhesion molecule belonging to the Ig-superfamily
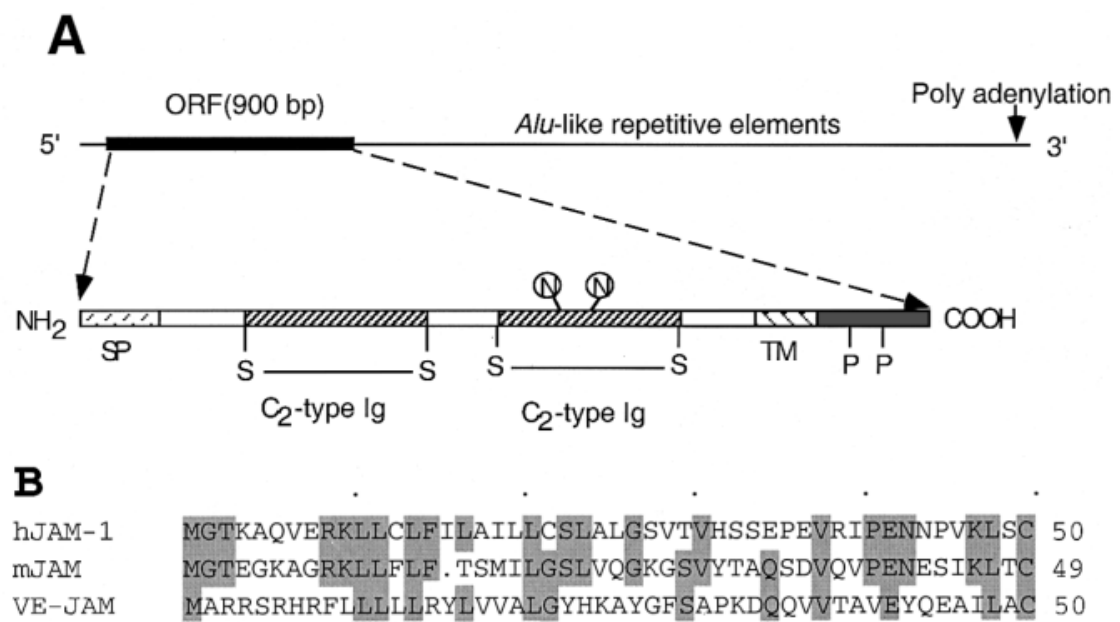

VE-JAM MARRSRHRFLLLLLRYLVVALGYHKAYGFSAPKDQQVVTAVEYQEAILAC 50

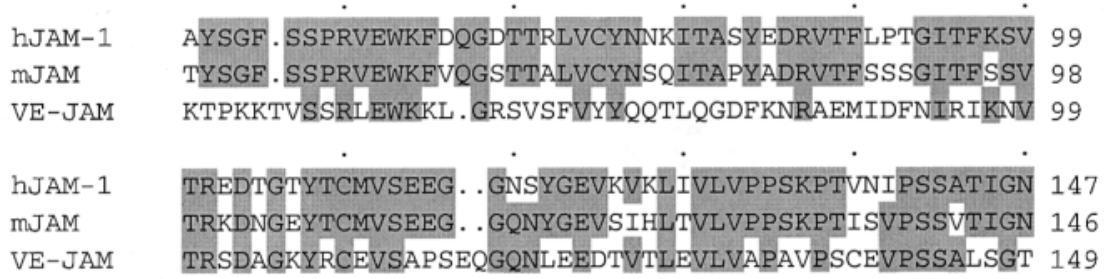

hJAM-1 RAVLTCSEQDGSPPSEYTWFKDGIVMPTNP. KSTRAFSNSSYVLNPTTGE 196 MJAM RAVLTCSEHDGSPPSEYSWFKDGISMLTADAKKTRAFMNSSFTIDPKSGD 196 VE-JAM VVELRCQDKEGNPAPEYTWFKDGIRLLENPRLGSQST.NSSYTMNTKTGT 198

hJAM-1 LVFDPLSASDTGEYSCEARNGYGTPMTSNAVRMEAVERNVGVIVAAVLVT 246 MJAM LIFDPVTAFDSGEYYCQAQNGYGTAMRSEAAHMDAVELNVGGIVAAVLVT 246 VE-JAM LQENTVSKLDTGEYSCEARNSVGYRRCPGK. RMQVDDLNISGIIAAVVVV 247

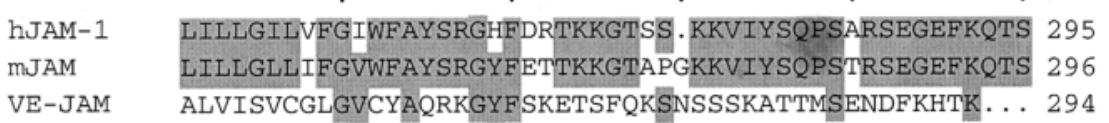

$\begin{array}{ll}\text { hJAM-1 } & \text { SFLV } 299 \\ \text { mJAM } & \text { SFLV } 300 \\ \text { VE-JAM } & \text { SEII } 298\end{array}$

Fig. 1. Sequence analysis of JAM-1. (A) cDNA and deduced protein domain structure of JAM-1. The 299 amino acid polypeptide precursor has a 27 amino acid signal peptide (SP), two C2-type Ig domains, a transmembrane domain (TM) and a 42 amino acid cytoplasmic domain. Two $\mathrm{N}$-glycosylation $(\mathrm{N})$ sites are present on the second $\mathrm{Ig}$ domain. Several serine/threonine protein kinase phosphorylation sites and two tyrosine phosphorylation sites $(\mathrm{P})$ are present in the cytoplasmic domain. (B) Deduced amino acid sequence comparison of JAM-1 with murine JAM and human VE-JAM. 


A
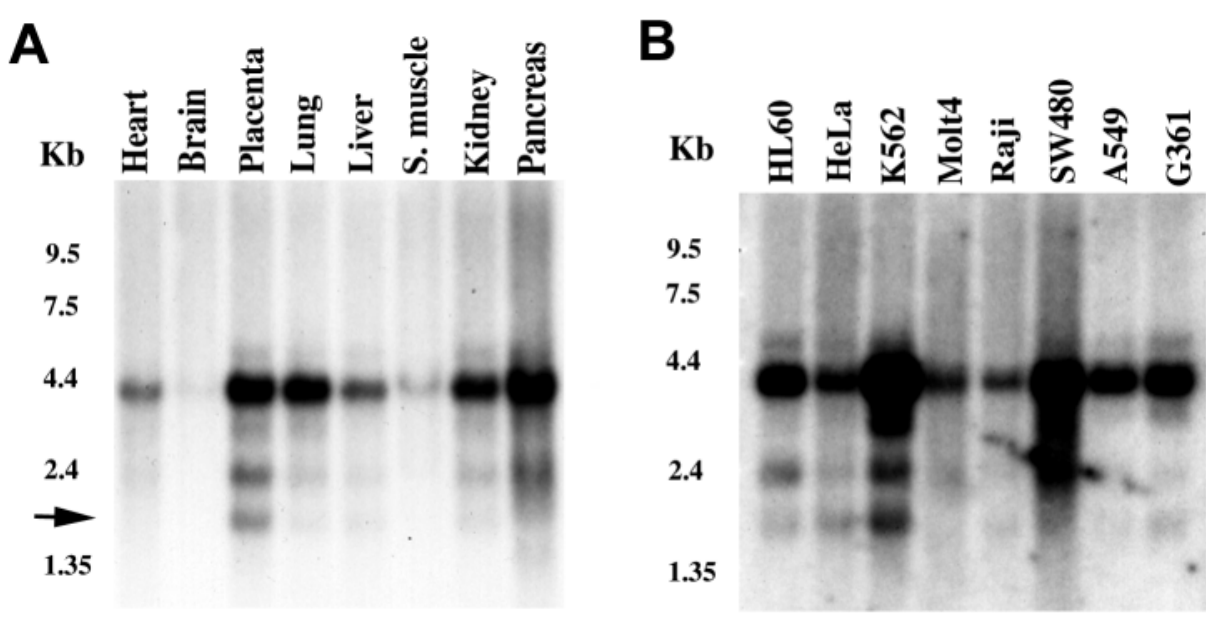

Fig. 2. Expression of JAM-1 mRNA in various human tissues and cell lines. Multiple tissue northern (MTN) blots of human tissues (A) and human cancer cell lines (B) were probed at high stringency using JAM-1 cDNA (coding region) as a probe. Three distinct species of message were detected, of which a $1.9 \mathrm{~kb}$ message corresponds to JAM-1

(arrow). The abundantly expressed $4.0 \mathrm{~kb}$ and less intense $2.4 \mathrm{~kb}$ bands are also visible. However, these bands are expressed in trace amounts in the brain. and having one amino acid residue less than JAM-1, has recently been cloned (Palmeri et al., 2000). VE-JAM has $\sim 32 \%$ identity to murine JAM or its human homolog JAM-1 (Fig. 1B). Recently, Cunningham et al. have cloned another member of the JAM family of cell adhesion molecules having $43 \%$ similarity to JAM-1 which maps to chromosome 21q21.2 (Cunningham et al., 2000). These results suggest that there is a family of cell adhesion molecules belonging to JAM-1.

\section{Expression of JAM-1}

Northern blot analysis performed to determine the expression pattern of JAM-1 indicates the presence of multiple specific bands indicative of closely related species or splicing variants of JAM-1 (Fig. 2A). The $1.9 \mathrm{~kb}$ transcript likely corresponds to the cloned JAM-1 species; however, two other major transcripts that were consistently seen even at high stringency, the 2.4 and $4.0 \mathrm{~kb}$ species, may represent JAM-1 molecular variants. The more abundant $4.0 \mathrm{~kb}$ transcript is expressed at high levels in the pancreas, placenta, lung, and kidney, and at low levels in the heart and liver. A similar pattern of expression was observed in various cancer cell lines, with the $4.0 \mathrm{~kb}$ species as the predominant transcript (Fig. 2B). It is thus possible that, like most of the CAMs, JAM-1 may also be expressed in different cells in different forms. Immunoblotting and immunohistological studies have shown that in addition to platelets and leukocytes, JAM-1 is also localized to the epithelial and endothelial cells of various tissues (Liu et al., 2000).

To determine if the larger transcripts of JAM-1 resulted from insertion of additional exons in the coding region of JAM-1, we performed RT-PCR analysis using both poly (A)+ RNA and total RNA isolated from K562 cells, which contain high levels of the larger transcripts. Fig. 3A shows the primers used to amplify various fragments of JAM-1 cDNA. Any insertion of exons occurring as a result of alternative splicing in either the coding region or in the 3 '-untranslated region (UTR) will produce an insert of larger than expected size. However, only expected-size products were amplified (Fig. 3B), suggesting that there may not be any insertions occurring in the coding region and in the $3^{\prime}$-UTR. These results suggest that the larger transcripts may have arisen from an extended $3^{\prime}$ or $5^{\prime}$-UTR.

\section{Isolation of full-length cDNA of JAM-1}

Unigene database search revealed several EST clones having large insert size. We obtained from ATCC an EST clone (AI923135) that had a $3.7 \mathrm{~kb}$ insert. Sequence analysis of this

A

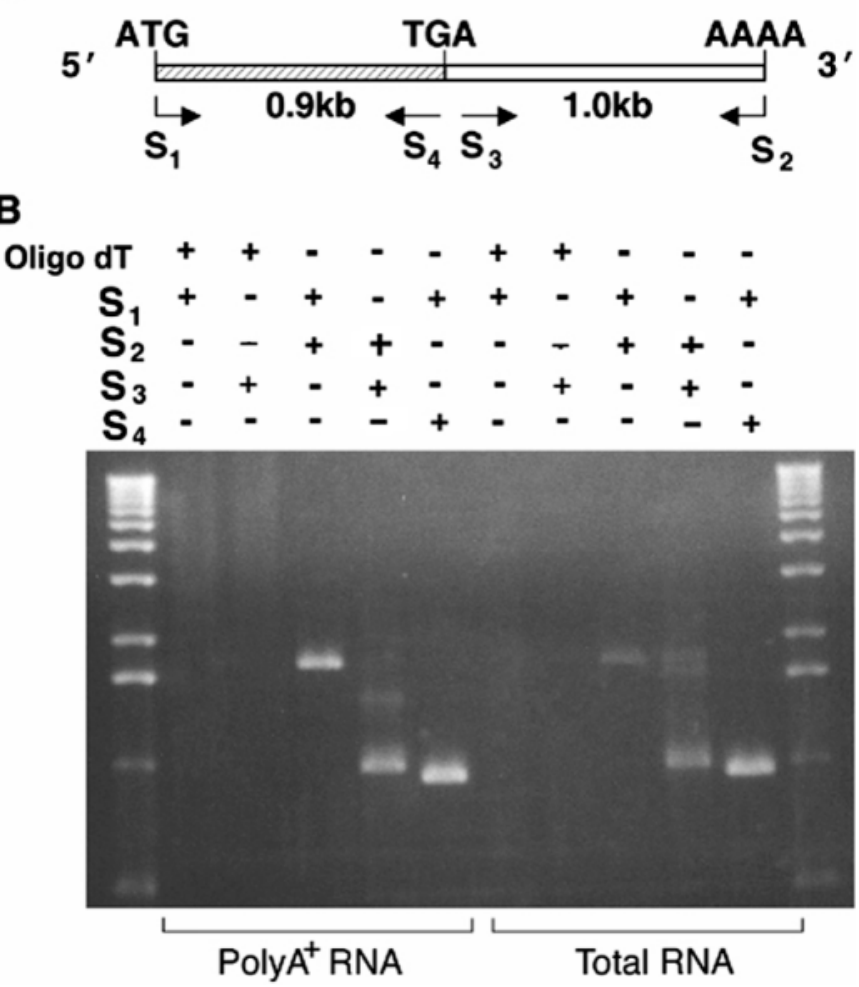

Fig. 3. RT-PCR amplification of JAM-1 transcripts from K562 cells. (A) Primers used in RT-PCR experiments with expected sizes of the products. Oligo dT and four specific primers - S1 and S3 (sense); S2 and S4 (antisense) derived from JAM-1 cDNA were used in PCR amplification. S1 and S2 should amplify $1.9 \mathrm{~kb}$ product, whereas S3 and $\mathrm{S} 2$, are expected to generate $1.0 \mathrm{~kb}$ product. S1 and S4 should produce $0.9 \mathrm{~kb}$ product. (B) Agarose gel showing the RT-PCRamplified products. PolyA ${ }^{+}$RNA (lanes 2-6), or Total RNA (lanes 711) prepared from K562 cells were used in RT-PCR reactions as described in Materials and Methods. Different sets of primers were used as indicated to amplify specific sizes of cDNA fragments. Lane 1 and lane 12 are molecular size markers. 
Fig. 4. Regional mapping of the JAM-1 gene by FISH Fluorescently labeled JAM-1 cDNA was hybridized to normal human lymphocyte chromosomes counterstained with DAPI. Biotinylated JAM-1 cDNA was detected with FITC-labeled avidin and anti-avidin. Separate images of metaphases (B) or partial spreads (C), and of the hybridization signal on chromosome 1 (arrow) were captured and overlaid electronically using a CCD-cooled camera with software from the IPLab Spectrum module (Scanalytics). The arrowheads in B indicate homologous pair of chromosome 1. The FISH signal localized the gene to 1q21.1-21.2. A Unigene database search revealed that the JAM- 1 gene is localized between microsatellite markers D1S2705 and D1S2768 (A).
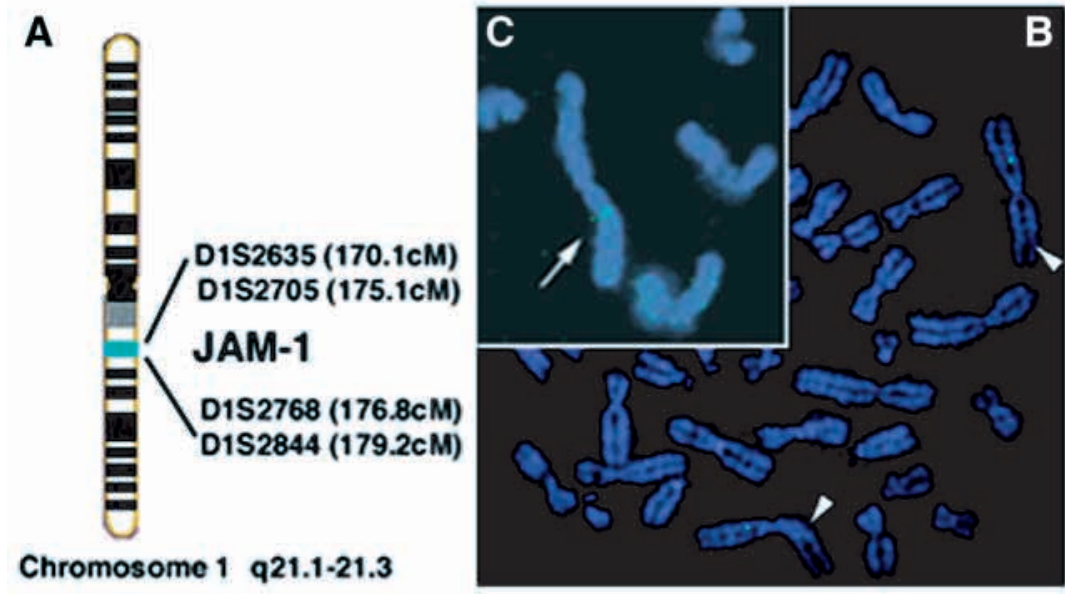

clone indicated that the JAM-1 message has a $2.7 \mathrm{~kb}$ long $3^{\prime}$ UTR that contains several repeats of Alu-like repetitive elements. The 3' end of this clone also has the polyadenylation signal that is not present in any of the earlier reports published on human JAM-1.

\section{Chromosomal localization of JAM-1}

To ascertain whether the different sized transcripts of JAM-1 originated from a single gene, the chromosomal localization of JAM-1 was determined using FISH (Deng et al., 1997; Nagle et al., 1994). In over 20 metaphases analyzed, FISH signals were exclusively detected on one or both homologs of chromosome 1, and DAPI-banding localized the gene to band q21.1 to q21.3 (Fig. 4). The low intensity of the signal could be due to the use of a cDNA fragment $(\sim 1.0 \mathrm{~kb})$ as a probe instead of genomic DNA. A Unigene search of the GenBank database further confirmed and narrowed the location of the gene for JAM-1 to a $1.7 \mathrm{cM}$ interval between the D1S2705 $(175.1 \mathrm{cM})$ and D1S2768 $(176.8 \mathrm{cM})$ microsetallite markers (http://www.ncbi.nlm.nih.gov/UniGene/clust.cgi?ORG=Hs\& $\mathrm{CID}=11482$ ). Together these results indicate that the observed multiple transcripts are the product of either a single gene or highly similar, tightly-linked genes. It should be noted that mutations responsible for several human genetic disorders map near this region of human chromosome 1, including Gaucher's disease, glaucoma, prostate cancer and Alzheimer's disease (http://www.ncbi.nlm.nih.gov/disease/chr1-4.html).

\section{Expression of recombinant JAM-1 in CHO cells and its cellular localization}

To determine the intracellular localization and function of JAM-1 more specifically, an HA epitope was engineered at the $\mathrm{N}$ terminus of JAM-1 between the signal peptide and the first Ig domain, and the resulting protein was expressed in $\mathrm{CHO}$ and T47D cells. The level of expression was monitored by western

Fig. 5. Surface expression of recombinant JAM-1. (A) CHO cells were transiently transfected with an expression vector producing HA-tagged JAM-1. 24 hours after transfection, cells were stained with an anti-HA antibody followed by a Texas Red-conjugated secondary. Cells were observed under confocal microscopy. JAM-1 localizes to the membrane of transfected CHO cells. (B) Stably transfected T47D cells were allowed to grow to confluency before fixing. JAM-1 localizes to the cell-cell junction. blotting using an anti-HA monoclonal antibody. As shown by confocal microscopy, recombinant JAM-1 transiently expressed in $\mathrm{CHO}$ cells localized to the cell membrane (Fig. $5 \mathrm{~A})$. Although JAM-1 is localized to the cell membrane, intense staining is observed at the cell-cell contacts formed by transfected cells (data not shown). In stably transfected confluent T47D cells, JAM-1 showed a cobblestone-like pattern of cellular localization specific to intercellular junctions (Fig. 5B). Since JAM-1 was also found to localize to membrane structures that resemble membrane ruffles in well-spread cells, the localization of JAM-1 (Fig. 6A) was compared with F-actin (Fig. 6B). JAM-1 was found to co-localize with F-actin at cellcell contacts and the membrane ruffles, but not at stress fibers (Fig. 6C). Fig. 6D is another representation of co-localization of JAM-1 with F-actin at the cell-cell junction.

\section{Homophilic interaction of JAM-1}

Because the most intense staining of JAM-1 was observed where two adjacent cells made contact with each other, this suggests that JAM-1 associates through homophilic interactions. To test this idea, $\mathrm{CHO}$ cells that were expressing high levels of JAM-1 were mixed with equal amounts of untransfected cells and plated on coverglass chambers. Untransfected cells did not show HA-tagged JAM-1 expression. In isolated cells, JAM-1 was found uniformly on the cell membrane. However, JAM-1 localizes only to the cellcell junctions formed by two transfected cells (Fig. 7A,B, bottom), and not to the cell-cell junctions formed by a transfected cell with an untransfected cell (Fig. 7B, top), suggesting that JAM-1 may facilitate cell adhesion through homophilic binding.
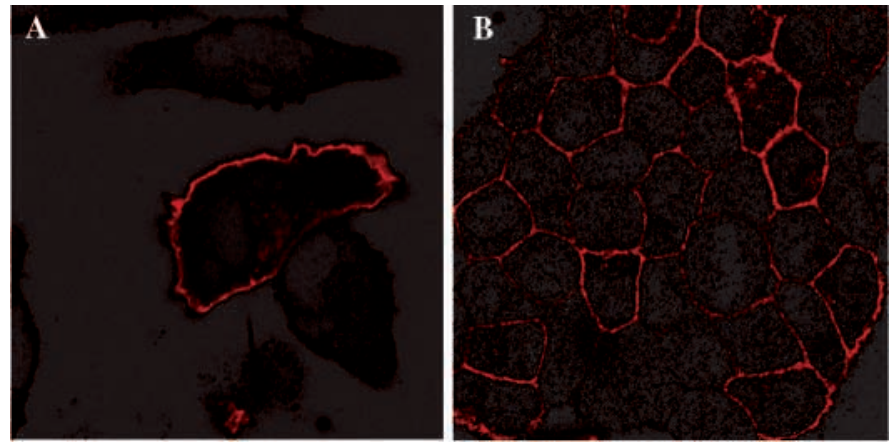
Fig. 6. Co-localization of JAM-1 with membrane ruffles at the cell-cell junction. $\mathrm{CHO}$ cells transfected with JAM-1 were stained with anti-HA antibody for JAM-1 and with FITC-labeled phalloidin for F-actin. (A) Intense JAM-1 staining is found at the cell-cell junctions (red).

(B) Phalloidin staining of F-actin stress fibers and membrane ruffles (green). (C) Merged image from A,B. JAM-1 colocalizes with F-actin at the cell-cell junction and membrane ruffles. However, it does not localize to the stress fibers. (D) Another representation of co-localization of JAM-1 and F-actin at cell-cell junctions.

\section{Adhesion of human platelets to JAM-1- expressing $\mathrm{CHO}$ cells}

To determine if JAM-1 facilitates platelet adhesion to the cells that express JAM-1, CHO cells that were mock transfected or transfected with JAM-1 were plated to form a confluent monolayer. Freshly isolated human platelets were allowed to bind to the monolayer, and bound platelets were visualized by using a fluorescently labeled cyclic RGD peptide that specifically binds to the platelet surface integrin $\alpha \operatorname{IIb} \beta 3$. Fewer than five platelets/field bound to the mock-transfected cells (Fig. 8B), whereas $\sim 35$ platelets/field, bound to the JAM-1-transfected cells (Fig. 8A). In three separate experiments, we found that about sixfold more platelets bound to $\mathrm{CHO}$ cells

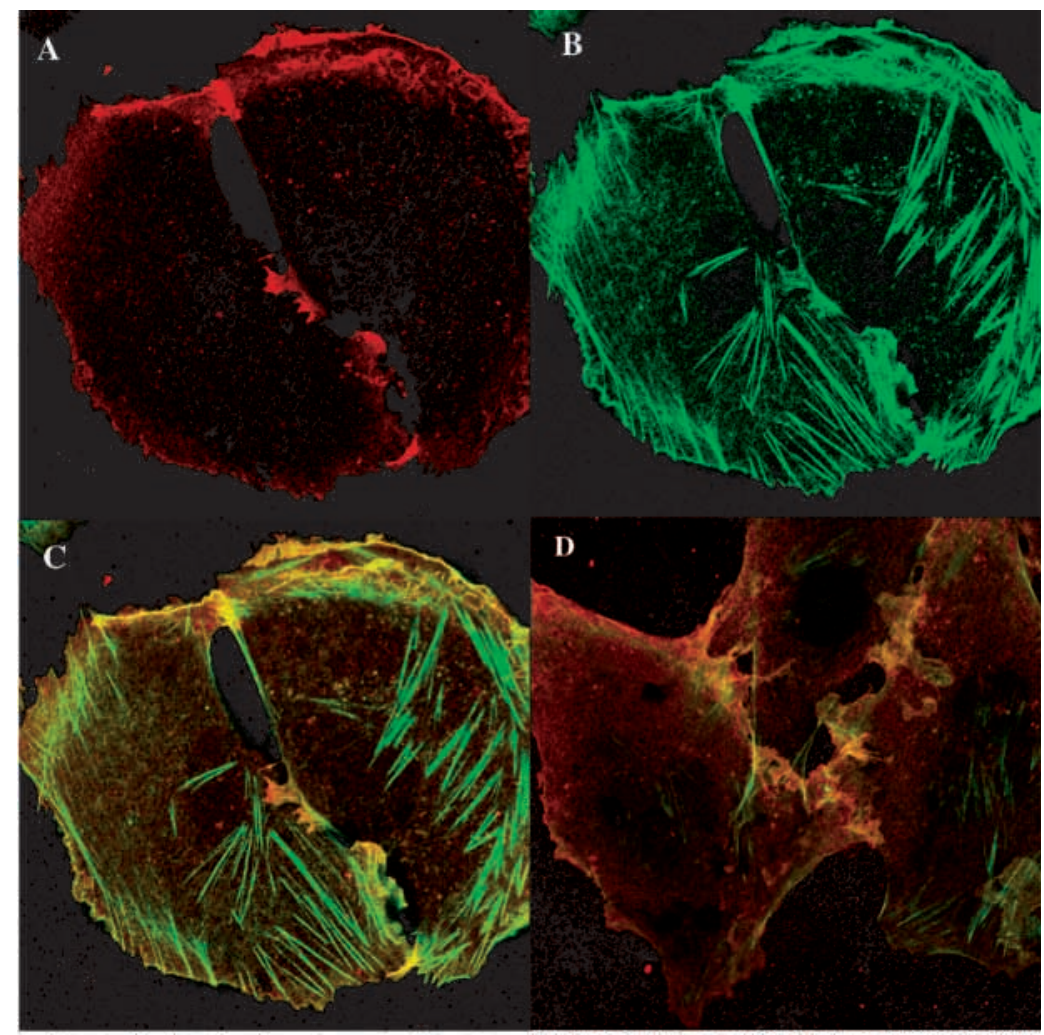
expressing JAM-1 than to non JAM-1-expressing cells (Fig. 8C). The increased binding of platelets to JAM-1-expressing cells was consistent with the level of JAM1 expression on the cell surface (data not shown). These results indicate that JAM-1 facilitates platelet adhesion to JAM-1expressing cell monolayers, probably through homophilic interactions.

\section{DISCUSSION}

Many platelet surface glycoproteins serve as antigens for autoantibodies (Beardsley, 1990; McMillan, 2000; Wadenvik et al., 1998). A number of monoclonal antibodies specific to these antigens stimulate platelets, leading to granular secretion and aggregation (Carroll et al., 1990; Jennings et al., 1985; Perutelli and Mori, 1993; Worthington et al., 1990). We have previously purified and characterized a novel platelet surface glycoprotein as an antigen for the stimulatory monoclonal antibody F11 (Naik et al., 1995). Molecular cloning of this surface antigen revealed that it is a novel type 1 membrane protein and a member of the Ig superfamily of cell adhesion molecules. Deduced amino acid sequence analysis indicates that it is $\sim 69 \%$ identical to the recently cloned murine junctional adhesion molecule, JAM (Martin-Padura et al., 1998), and that it is identical to the human homolog of JAM (Ozaki et al., 1999; Williams et al., 1999). Recently, another group independently reported the cloning of the platelet F11 receptor, F11R, which is identical to our JAM-1 (Sobocka et al., 2000). Sequence data of each of these proteins have been deposited in the database as separate entries under separate names (JAM-1, AF111713; F11R, AF207907). Very recently, two Ig-family members named VE-JAM and JAM-2 have been identified (Palmeri et al., 2000). Although similar in size and

in their intracellular distribution, these newly identified members have different tissue distributions than JAM-1. VEJAM is not expressed on platelets and leukocytes whereas, JAM-2 is predominantly expressed in the heart (Palmeri et al., 2000). Interestingly, JAM-2 and VE-JAM are distinct products of genes mapped to the same locus on chromosome 21 (21q21.2), in contrast to JAM-1, which is localized to chromosome 1.

It has been reported that JAM-1 has two V-type Ig domains and hence may belong to a novel family of cell adhesion molecules (Ozaki et al., 1999; Williams et al., 1999). However, Sobocka et al. predicted the presence of two C2-type domains (Sobocka et al., 2000). Our sequence analysis supports the prediction of two C2-type domains based on the classification of Ig-domains (Williams, 1987; Williams and Barclay, 1988). The main structural difference between V-type and C2-type domains is that the V-domain has an extra loop in the middle, loop D that links E and F strands (Harpaz and Chothia, 1994; Williams, 1987; Williams and Barclay, 1988). Consequently, V-type and C2-type domains have different lengths between the cysteine residues that forms the disulfide bridges. The Vtype domains usually have 65-75 amino acid residues, whereas C2-type domains have 55-60 residues (Harpaz and Chothia, 1994).

Amino acid sequence analysis indicates the presence of two putative $\mathrm{N}$-glycanase sites in the second Ig domain of the extracellular domain of JAM-1. This is in contrast to the findings of a single $\mathrm{N}$-glycosylation site in previous reports (Ozaki et al., 1999; Sobocka et al., 2000). The presence of two glycosylation sites supports our previous finding that F11 recognizes a doublet of 35 and $32 \mathrm{kDa}$ in platelet extract that was converted into a single $29 \mathrm{kDa}$ core protein upon $\mathrm{N}$ - 

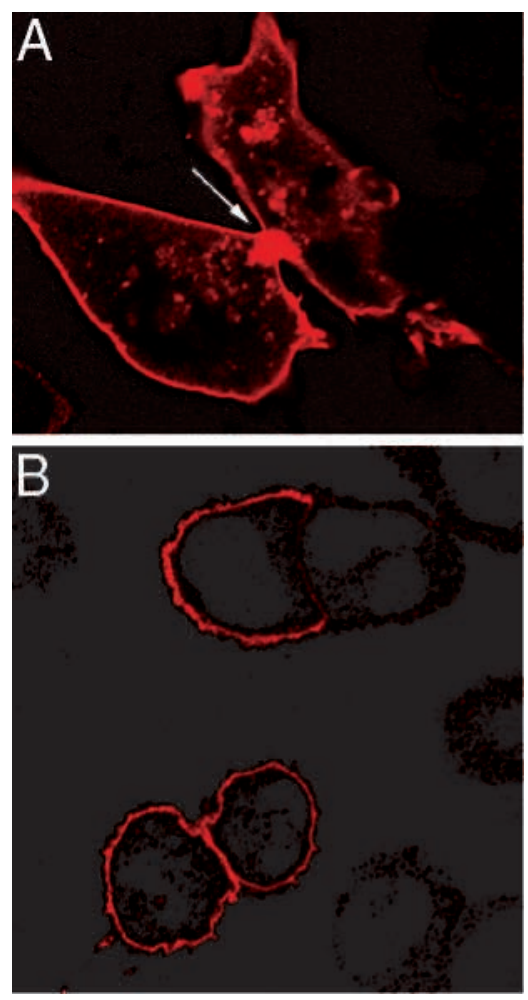

Fig. 7. Homophilic interaction of JAM-1 in CHO cells. Untransfected $\mathrm{CHO}$ cells and $\mathrm{CHO}$ cells expressing HA-tagged JAM-1 were stained with an anti-HA antibody as in Fig. 5. Two JAM-1-expressing cells forming a contact are shown in A. (B) A JAM-1-expressing cell either in contact with another cell that expresses JAM-1, or in contact with an untransfected cell. Intense staining is observed at the place where two adjacent cells actually make contact with each other (A). JAM-1 localizes to the cell-cell contact formed by two cells that express JAM-1 (B, bottom) and is not localized to the junction formed by a JAM-1-expressing cell with an untransfected cell (B, top).

glycanase treatment (Naik et al., 1995). Since the molecular mass of a single $\mathrm{N}$-linked sugar moiety is generally of the order of 3-4 $\mathrm{kDa}$ (Scott et al., 1989), it is possible that the $35 \mathrm{kDa}$ form has two such sugar groups, and the $32 \mathrm{kDa}$ form has one.
The cytoplasmic domain of JAM-1 has several putative phosphorylation sites for PKC and other protein kinases. This is in agreement with our previous finding that JAM-1 is phosphorylated in vitro and in vivo by PKC (Naik et al., 1995), which has recently been confirmed by Ozaki et al. (Ozaki et al., 2000). Other structural features of JAM-1 include two tyrosine residues in a context similar to the cytoplasmic domain of PDGFR, which is capable of binding to SH2 domain-containing proteins (Jackson et al., 1997b). These two tyrosines are also conserved in mouse JAM. Interestingly, similar tyrosine residues are also present in the cytoplasmic domain of PECAM-1, a member of the CAM family of proteins that has been shown to bind to the SH2-containing protein, SHP2P (Jackson et al., 1997b). PECAM-1 is also expressed on platelet and endothelial cells and is involved in platelet activation upon antibody crosslinking (Varon et al., 1998). The cytoplasmic domain of JAM-1 has a conserved motif (S/TXXV) that is capable of binding PDZ (PSD-95, discs large, ZO-1) domains. In fact, JAM-1 has recently been found to bind to two tight-junction proteins, ZO-1 and AF-6 through their PDZ domains (Ebnet et al., 2000).

Structurally, JAM-1 resembles other cell adhesion molecules (CAMs), which have a large extracellular domain containing multiple Ig-like subdomains and a short cytoplasmic domain. Several members of the Ig-superfamily have been shown to be expressed in multiple forms, either as a result of alternate splicing or as products of closely related genes (Baldwin et al., 1994; Rahuel et al., 1996; Walsh and Doherty, 1997). For example, PECAM-1 is expressed in different spliced forms (Baldwin et al., 1994; Buck et al., 1993), whereas ICAM family members ICAM-1, ICAM-2, and ICAM-3, are all derived from separate genes displaying significant sequence homology (de Fougerolles and Springer, 1992; Fawcett et al., 1992; Vazeux et al., 1992; Wuthrich, 1992). In contrast to murine JAM (Martin-Padura et al., 1998), multiple forms of the human JAM-1 mRNA are expressed. The physical mapping of JAM-1 to the long arm of chromosome 1 excludes the possibility that JAM-1 gene products arise from homologous genes dispersed throughout the genome. RT-PCR data suggest that the coding region of JAM-1 does not have insertions resulting from alternative splicing. Interestingly, the VE-JAM message is also expressed in three forms $(4.4 \mathrm{~kb}, 1.4 \mathrm{~kb}$ and
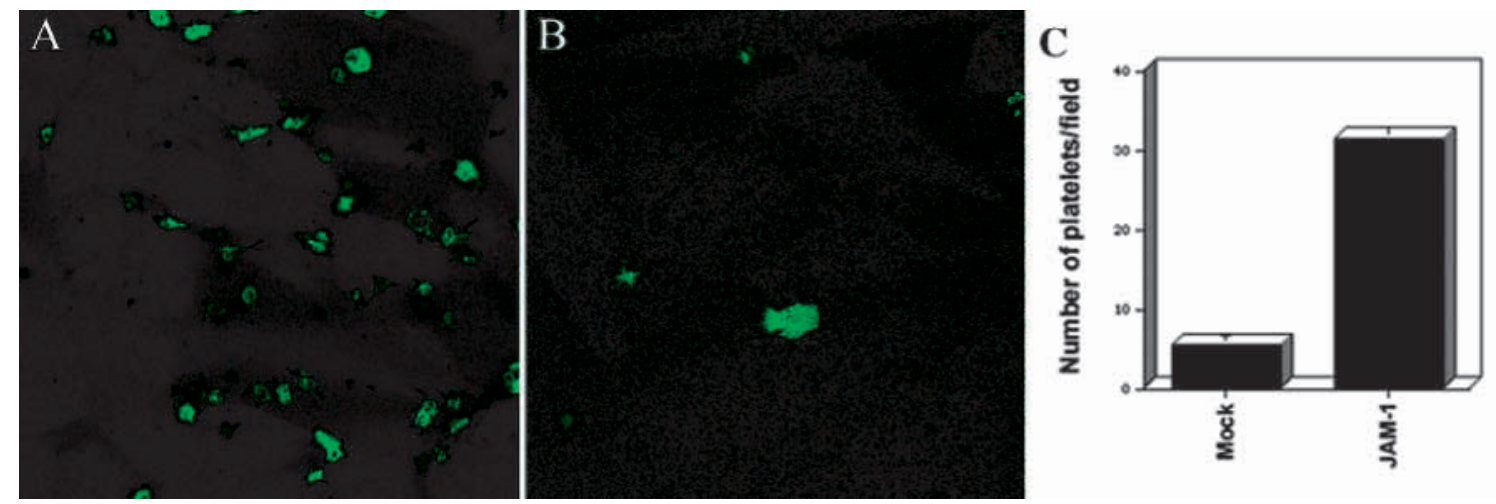

Fig. 8. Platelets adhere to CHO cells expressing JAM-1. Human platelets from PRP were allowed to adhere to JAM-1-transfected (A) and mock-transfected (B) CHO cells, which were grown in coverglass chambers to confluency. The bound platelets were fluorescently detected as described in the experimental procedures. Platelets bound per field (100× oil immerson objective) were counted randomly. (C) Results of three separate experiments presented as mean \pm s.e.m. 
$1.2 \mathrm{~kb}$ ), with the $\sim 4.4 \mathrm{~kb}$ transcript being most abundant (Palmeri et al., 2000). The multiple species of JAM-1 message results from the different sizes of the $3^{\prime}$-UTR formed as a result of insertion of $A l u$-like repetitive elements.

Platelets have been shown to express JAM- 1 constitutively on their surface. Adhesion of platelets to a monolayer of $\mathrm{CHO}$ cells expressing JAM-1 on their surface but not to the mocktransfected cells suggests that JAM-1 may facilitate cell-cell adhesion through homophilic interactions. Ozaki et al. have shown that the combined treatment of endothelial cells with proinflammatory cytokines, TNF- $\alpha$ and IFN- $\gamma$, causes the disappearance of JAM-1 from the intracellular junctions and its redistribution on the cell surface (Ozaki et al., 1999). It is thus possible that the JAM-1 exposed on the activated endothelial cell surface may facilitate the interaction of circulating leukocytes and platelets with the endothelium. However, the JAM-1-specific mAb failed to inhibit leukocyte transmigration through a monolayer of endothelial cells (data not shown). It is possible that our anti-JAM-1 mAb may not be a function-blocking antibody. Antibody against murine JAM has been reported to block leukocyte transmigration into the CNS induced by cytokines (Del Maschio et al., 1999). Although our results indicate that JAM-1 is capable of homophilic interactions, its heterophilic interactions with other cell-surface proteins not expressed in $\mathrm{CHO}$ cells cannot be excluded. Several members of the CAM family are known to interact by both homophilic and heterophilic interactions (Muller, 1995; Wu and Lian, 1997).

Like most of the known stimulatory monoclonal antibodies, platelet stimulation by $\mathrm{F} 11$ requires binding to its antigen, JAM-1, through its Fab portion, and to the FcyRII receptor through its Fc portion (Naik et al., 1995). This receptor crosslinking is necessary for the full platelet activation response. However, in the absence of Fc receptor crosslinking, F11 is capable of potentiating the responses of suboptimal stimuli of physiological agonists such as ADP and thrombin. This crosstalk between different signaling pathways suggests that the cytoplasmic tails of the respective membrane receptors may mediate the stimulation of common intracellular regulatory circuitry. We have previously shown that JAM-1 is phosphorylated as a result of antibody binding (Naik et al., 1995). It is therefore possible that JAM-1 may be involved in transmembrane signaling. Further characterization of the signaling pathway induced by JAM-1 is in progress.

It has now been demonstrated that Ig-like CAMs and selectins on endothelial cells also function as outside-in signaling receptors (Gurubhagavatula et al., 1998; Suter et al., 1998). The signaling events induced by these CAMs include a rise in intracellular $\mathrm{Ca}^{2+}$, protein phosphorylation and cytoskeletal rearrangements. It has also been shown that platelet-endothelial cell adhesion molecule 1 (PECAM-1) becomes phosphorylated and associates with the platelet cytoskeleton upon platelet activation (Newman et al., 1992). Our previous finding of JAM-1 as the co-receptor for a plateletactivating antibody strongly suggests that JAM-1, in addition to cell-cell adhesion, may also be involved in intracellular signaling. Thus, it may appear that this protein has two independent functions: one structural, which is related to the formation of tight junctions through homophilic interactions, and the other related to signal transduction through heterophilic interactions. These two distinct functions may be cell specific.
Although JAM-1 may serve as an antigen on the plasma membrane for autoantibodies during certain autoimmune disorders, its physiological ligand on other cells is yet to be identified. The identification of this physiological ligand will help to define the physiological function of this molecule.

The authors thank Leslie Parise and Beverly Mitchel at the University of North Carolina at Chapel Hill for providing support during the early stages of this project. We also thank Melinda Duncan for useful comments and for critically reading the manuscript. This work was supported by Grants (UPN) from the National Institutes of Health HL57630, HL63960 and the American Heart AssociationPennsylvania \& Delaware Affiliate. Part of this work was presented at the XVIIth Congress of the International Society on Thrombosis and Hemostasis, Washington DC, August 14-21, 1999.

\section{REFERENCES}

Baldwin, H. S., Shen, H. M., Yan, H. C., DeLisser, H. M., Chung, A., Mickanin, C., Trask, T., Kirschbaum, N. E., Newman, P. J., Albelda, S. M. et al. (1994). Platelet endothelial cell adhesion molecule-1 (PECAM1/CD31): alternatively spliced, functionally distinct isoforms expressed during mammalian cardiovascular development. Development 120, 25392553.

Beardsley, D. S. (1990). Platelet membrane glycoproteins: role in primary hemostasis and component antigens. Yale J. Biol. Med. 63, 469-475.

Buck, C. A., Baldwin, H. S., DeLisser, H., Mickanin, C., Shen, H. M., Kennedy, G., Chen, A., Edelman, J. M. and Albelda, S. M. (1993). Cell adhesion receptors and early mammalian heart development: an overview. C R Acad. Sci. III 316, 838-859.

Carroll, R. C., Worthington, R. E. and Boucheix, C. (1990). Stimulusresponse coupling in human platelets activated by monoclonal antibodies to the CD9 antigen, a $24 \mathrm{kDa}$ surface-membrane glycoprotein. Biochem. J. 266, 527-535.

Celi, A., Lorenzet, R., Furie, B. and Furie, B. C. (1997). Platelet-leukocyteendothelial cell interaction on the blood vessel wall. Semin. Hematol. 34, 327-335.

Chiba, R., Nakagawa, N., Kurasawa, K., Tanaka, Y., Saito, Y. and Iwamoto, I. (1999). Ligation of CD31 (PECAM-1) on endothelial cells increases adhesive function of alphavbeta3 integrin and enhances beta1 integrin-mediated adhesion of eosinophils to endothelial cells. Blood 94, 1319-1329.

Cunningham, S. A., Arrate, M. P., Rodriguez, J. M., Bjercke, R. J., Vanderslice, P., Morris, A. P. and Brock, T. A. (2000). A novel protein with homology to the junctional adhesion molecule. Characterization of leukocyte interactions. J. Biol. Chem. 275, 34750-34756.

de Fougerolles, A. R. and Springer, T. A. (1992). Intercellular adhesion molecule 3, a third adhesion counter-receptor for lymphocyte functionassociated molecule 1 on resting lymphocytes. J. Exp. Med. 175, 185-190.

Dejana, E., Corada, M. and Lampugnani, M. G. (1995). Endothelial cellto-cell junctions. FASEB J. 9, 910-918.

Del Maschio, A., De Luigi, A., Martin-Padura, I., Brockhaus, M., Bartfai, T., Fruscella, P., Adorini, L., Martino, G., Furlan, R., De Simoni, M. G. et al. (1999). Leukocyte recruitment in the cerebrospinal fluid of mice with experimental meningitis is inhibited by an antibody to junctional adhesion molecule (JAM). J. Exp. Med. 190, 1351-1356.

Deng, X., Moran, J., Copeland, N. G., Gilbert, D. J., Jenkins, N. A., Primakoff, P. and Martin-DeLeon, P. A. (1997). The mouse Spam1 maps to proximal chromosome 6 and is a candidate for the sperm dysfunction in $\mathrm{Rb}(6.16) 24 \mathrm{Lub}$ and $\mathrm{Rb}(6.15) 1$ Ald heterozygotes. Mamm. Genome 8, 94-97.

Ebnet, K., Schulz, C. U., Meyer Zu Brickwedde, M. K., Pendl, G. G. and Vestweber, D. (2000). Junctional Adhesion Molecule (JAM) interacts with the PDZ domain containing proteins AF-6 and ZO-1. J. Biol. Chem. (in press).

Etzioni, A. (1996). Adhesion molecules-their role in health and disease. Pediatr. Res. 39, 191-198.

Fawcett, J., Holness, C. L., Needham, L. A., Turley, H., Gatter, K. C., Mason, D. Y. and Simmons, D. L. (1992). Molecular cloning of ICAM-3, a third ligand for LFA-1, constitutively expressed on resting leukocytes. Nature 360, 481-484. 
Gurubhagavatula, I., Amrani, Y., Pratico, D., Ruberg, F. L., Albelda, S. M. and Panettieri, R. A., Jr. (1998). Engagement of human PECAM-1 (CD31) on human endothelial cells increases intracellular calcium ion concentration and stimulates prostacyclin release. J. Clin. Invest. 101, 212222

Harpaz, Y. and Chothia, C. (1994). Many of the immunoglobulin superfamily domains in cell adhesion molecules and surface receptors belong to a new structural set which is close to that containing variable domains. J. Mol. Biol. 238, 528-539.

Jackson, D. E., Loo, R. O., Holyst, M. T. and Newman, P. J. (1997a). Identification and characterization of functional cation coordination sites in platelet endothelial cell adhesion molecule-1. Biochemistry 36, 93959404.

Jackson, D. E., Ward, C. M., Wang, R. and Newman, P. J. (1997b). The protein-tyrosine phosphatase SHP-2 binds platelet/endothelial cell adhesion molecule-1 (PECAM-1) and forms a distinct signaling complex during platelet aggregation. Evidence for a mechanistic link between PECAM-1- and integrin-mediated cellular signaling. J. Biol. Chem. 272, 6986-6993.

Jennings, L. K., Phillips, D. R. and Walker, W. S. (1985). Monoclonal antibodies to human platelet glycoprotein IIb beta that initiate distinct platelet responses. Blood 65, 1112-1119.

Kornecki, E., Walkowiak, B., Naik, U. P. and Ehrlich, Y. H. (1990). Activation of human platelets by a stimulatory monoclonal antibody. J. Biol. Chem. 265, 10042-10048.

Liu, Y., Nusrat, A., Schnell, F. J., Reaves, T. A., Walsh, S., Pochet, M. and Parkos, C. A. (2000). Human junction adhesion molecule regulates tight junction resealing in epithelia. J. Cell Sci. 113, 2363-2374.

Martin-Padura, I., Lostaglio, S., Schneemann, M., Williams, L., Romano, M., Fruscella, P., Panzeri, C., Stoppacciaro, A., Ruco, L., Villa, A. et al. (1998). Junctional adhesion molecule, a novel member of the immunoglobulin superfamily that distributes at intercellular junctions and modulates monocyte transmigration. J. Cell Biol. 142, 117-127.

McMillan, R. (2000). The pathogenesis of chronic immune (idiopathic) thrombocytopenic purpura. Semin. Hematol. 37, 5-9.

Mousa, S., Bozarth, J., Naik, U. and Slee, A. (2000). Platelet GPIIb/IIIa binding characteristics of small molecule RGD mimetics: Distinct binding profile for Roxifiban. Br. J. Pharmacol. (in press).

Muller, W. A. (1995). The role of PECAM-1 (CD31) in leukocyte emigration: studies in vitro and in vivo. J. Leukocyte Biol. 57, 523-528.

Nagle, D. L., Martin-DeLeon, P., Hough, R. B. and Bucan, M. (1994), Structural analysis of chromosomal rearrangements associated with the developmental mutations $\mathrm{Ph}, \mathrm{W} 19 \mathrm{H}$, and $\mathrm{Rw}$ on mouse chromosome 5. Proc. Natl. Acad. Sci. USA 91, 7237-7241.

Naik, U. P., Kornecki, E. and Ehrlich, Y. H. (1991). Phosphorylation and dephosphorylation of human platelet surface proteins by an ecto-protein kinase/phosphatase system. Biochim. Biophys. Acta 1092, 256-264.

Naik, U. P., Ehrlich, Y. H. and Kornecki, E. (1995). Mechanisms of platelet activation by a stimulatory antibody: cross-linking of a novel platelet receptor for monoclonal antibody F11 with the FcyRII receptor. Biochem. J. 310, 155-162.

Newman, P. J., Hillery, C. A., Albrecht, R., Parise, L. V., Berndt, M. C., Mazurov, A. V., Dunlop, L. C., Zhang, J. and Rittenhouse, S. E. (1992). Activation-dependent changes in human platelet PECAM-1: phosphorylation, cytoskeletal association, and surface membrane redistribution. J. Cell Biol. 119, 239-246.

Ozaki, H., Ishii, K., Horiuchi, H., Arai, H., Kawamoto, T., Okawa, K., Iwamatsu, A. and Kita, T. (1999). Cutting edge: combined treatment of TNF-alpha and IFN-gamma causes redistribution of junctional adhesion molecule in human endothelial cells. J. Immunol. 163, 553-557.

Ozaki, H., Ishii, K., Arai, H., Horiuchi, H., Kawamoto, T., Suzuki, H. and Kita, T. (2000). Junctional adhesion molecule (JAM) is phosphorylated by protein kinase $\mathrm{C}$ upon platelet activation. Biochem. Biophys. Res. Commun. 276, 873-878.

Palmeri, D., van Zante, A., Huang, C. C., Hemmerich, S. and Rosen, S. D. (2000). Vascular endothelial junction-associated molecule, a novel member of the immunoglobulin superfamily, is localized to intercellular boundaries of endothelial cells. J. Biol. Chem. 275, 19139-19145.
Perutelli, P. and Mori, P. G. (1993). Activation of human platelets by monoclonal antibodies. Haematologica 78, 172-177.

Rahuel, C., Le Van Kim, C., Mattei, M. G., Cartron, J. P. and Colin, Y. (1996). A unique gene encodes spliceoforms of the B-cell adhesion molecule cell surface glycoprotein of epithelial cancer and of the Lutheran blood group glycoprotein. Blood 88, 1865-1872.

Rosales, C., O'Brien, V., Kornberg, L. and Juliano, R. (1995). Signal transduction by cell adhesion receptors. Biochim. Biophys. Acta 1242, 7798.

Scott, J. L., Dunn, S. M., Jin, B., Hillam, A. J., Walton, S., Berndt, M. C., Murray, A. W., Krissansen, G. W. and Burns, G. F. (1989). Characterization of a novel membrane glycoprotein involved in platelet activation. J. Biol. Chem. 264, 13475-13482.

Sobocka, M. B., Sobocki, T., Banerjee, P., Weiss, C., Rushbrook, J. I., Norin, A. J., Hartwig, J., Salifu, M. O., Markell, M. S., Babinska, A. et al. (2000). Cloning of the human platelet F11 receptor: a cell adhesion molecule member of the immunoglobulin superfamily involved in platelet aggregation. Blood 95, 2600-2609.

Spychala, J., Datta, N. S., Takabayashi, K., Datta, M., Fox, I. H., Gribbin, T. and Mitchell, B. S. (1996). Cloning of human adenosine kinase cDNA: sequence similarity to microbial ribokinases and fructokinases. Proc. Natl. Acad. Sci. USA 93, 1232-1237.

Suter, D. M., Errante, L. D., Belotserkovsky, V. and Forscher, P. (1998). The Ig superfamily cell adhesion molecule, apCAM, mediates growth cone steering by substrate-cytoskeletal coupling. J. Cell Biol. 141, 227-240.

Takaishi, K., Sasaki, T., Kotani, H., Nishioka, H. and Takai, Y. (1997). Regulation of cell-cell adhesion by rac and rho small G proteins in MDCK cells. J. Cell Biol. 139, 1047-1059.

Vaporciyan, A. A., DeLisser, H. M., Yan, H. C., Mendiguren, II, Thom, S. R., Jones, M. L., Ward, P. A. and Albelda, S. M. (1993). Involvement of platelet-endothelial cell adhesion molecule-1 in neutrophil recruitment in vivo. Science 262, 1580-1582.

Varon, D., Jackson, D. E., Shenkman, B., Dardik, R., Tamarin, I., Savion, N. and Newman, P. J. (1998). Platelet/endothelial cell adhesion molecule1 serves as a costimulatory agonist receptor that modulates integrindependent adhesion and aggregation of human platelets. Blood 91, 500-507.

Vazeux, R., Hoffman, P. A., Tomita, J. K., Dickinson, E. S., Jasman, R. L., St. John, T. and Gallatin, W. M. (1992). Cloning and characterization of a new intercellular adhesion molecule ICAM-R. Nature 360, 485-488.

Wadenvik, H., Stockelberg, D. and Hou, M. (1998). Platelet proteins as autoantibody targets in idiopathic thrombocytopenic purpura. Acta Paediatr. Suppl. 424, 26-36.

Wahl, S. M., Feldman, G. M. and McCarthy, J. B. (1996). Regulation of leukocyte adhesion and signaling in inflammation and disease. J. Leukocyte Biol. 59, 789-796.

Walsh, F. S. and Doherty, P. (1997). Neural cell adhesion molecules of the immunoglobulin superfamily: role in axon growth and guidance. Аппи. Rev. Cell Dev. Biol. 13, 425-456.

Wang, F., Naik, U. P., Ehrlich, Y. H., Osada, S., Ohno, S. and Kornecki, E. (1995). Stimulatory antibody-induced activation and selective translocation of protein kinase $\mathrm{C}$ isoenzymes in human platelets. Biochem. J. 311, 401-406.

Williams, A. F. (1987). A year in the life of the immunoglobulin superfamily. Immunol. Today 8, 298-303.

Williams, A. F. and Barclay, A. N. (1988). The Immunoglobulin superfamilydomains for surface recognition. Annu. Rev. immunol. 6, 381-405.

Williams, L. A., Martin-Padura, I., Dejana, E., Hogg, N. and Simmons, D. L. (1999). Identification and characterisation of human junctional adhesion molecule (JAM). Mol. Immunol. 36, 1175-1188.

Worthington, R. E., Carroll, R. C. and Boucheix, C. (1990). Platelet activation by $\mathrm{CD} 9$ monoclonal antibodies is mediated by the Fc gamma II receptor. Br. J. Haematol. 74, 216-222.

Wu, X. W. and Lian, E. C. (1997). Binding properties and inhibition of platelet aggregation by a monoclonal antibody to CD31 (PECAM-1). Arterioscler. Thromb. Vasc. Biol. 17, 3154-3158.

Wuthrich, R. P. (1992). Monoclonal antibodies targeting murine LFA-1 induce LFA-1/ICAM-1-independent homotypic lymphocyte aggregation. Cell Immunol. 144, 22-31. 